Supporting information for:

\title{
Blue Photoluminescence from Hyperbranched Poly(amino ester)s
}

\author{
Decheng Wu, ${ }^{\dagger, \ddagger}$ Ye Liu, ${ }^{*, \dagger}$ Chaobin He, ${ }^{\dagger}$ Suat Hong Goh $^{\ddagger}$ \\ Institute of Materials Research and Engineering, 3 Research Link, Singapore 117602, Department \\ of Chemistry, National University of Singapore, 3 Science Drive 3, Singapore 117543
}

\section{Experimental Part}

\section{Characterization}

${ }^{1} \mathrm{H}$ NMR and ${ }^{13} \mathrm{C}$ NMR studies were performed on a Bruker DRX-400 spectrometer. Gel permeation chromatography (GPC) was implemented on a Waters 2690 apparatus using Waters Styragel HR 1 and 5E columns with a Waters 410 refractive index detector and a miniDAWN light scattering detector (Wyatt Technology). 0.1 M piperidine/THF was adopted as eluent. UV-vis spectra were recorded on a Shimadzu 3101 PC spectrometer, and fuorescence measurement was carried out on a Perkin-Elmer LS 50B photoluminescence spectrometer with a xenon lamp as a light source.

Brookhaven BI-9000AT Digital Autocorrelator was used for dynamic light scattering measurements. The scattering angle was fixed at $90^{\circ}$ for measuring hydrodynamic radius $\left(\mathrm{R}_{\mathrm{h}}\right) . \mathrm{R}_{\mathrm{h}}$ and polydispersity (PDI), i.e., $<\mu_{2}>/ \Gamma^{2}$, were obtained using a cumulant analysis. Radius of gyration $\left(\mathrm{R}_{\mathrm{g}}\right)$ was measured using small angle X-ray scattering (SAXS). SAXS measurements were performed on a SAXS system (Bruker AXS Inc. NanoStar SAXS) with a pinhole collimation configuration and a $\mathrm{CuK} \alpha(\lambda=1.5418 \AA \dot{A})$ radiation source. SAXS signal was detected using a two-dimensional multi-wire proportional HI-STAR area detector corrected for dark current, empty beam, solution cell and solvent contributions. Samples were scanned at a voltage of $40 \mathrm{kV}$ and a current of $35 \mathrm{~mA}$. Scattering intensity $I(\mathrm{q})$ and corresponding wave vector $q$ were collected for analysis, 
where $q=4 \pi \sin \theta / \lambda, \theta$ and $\lambda$ are the scattering angle and the wavelength of $\mathrm{x}$-ray, respectively. Fluorescence lifetimes were obtained using Nd:YAG laser and optical parametric oscillator (OPO) system (Spectra-Physics).

\section{Materials}

1,4-Butanediol diacrylate (BDA, 90\%), 1-(2-aminoethyl) piperazine (AEPZ, 99\%), 2amino ethanol (AEOL, 99+\%), 3-amino-1,2-propanediol (APDIOL, 97\%), deuterium chloroform, potassium iodide (KI, 99\%), deuterium dimethyl sulfoxide (DMSO- $\mathrm{d}_{6}$ ), and DMSO (99.9\%) were purchased from Aldrich, piperidine (99\%) from Lancaster, chloroform and diethyl ether were from Tedia, and polyethylenimine (PEI, MW 25,000) from Sigma Chemical Co. (St. Louis, MO), respectively. DMSO was dried using molecular sieves, BDA was purified by vacuum distillation $\left(90{ }^{\circ} \mathrm{C} / 50 \mathrm{~Pa}\right)$ in the presence of a little amount of hydroquinone, and other reagents were used as received.

\section{Synthesis of hyperbranched poly(BDA2-AEPZ1)-vinyl}

In a typical process, $11.4 \mathrm{mmol}$ of BDA was added into a solution of $5.7 \mathrm{mmol}$ of AEPZ in DMSO $(15 \mathrm{ml})$. The polymerization was performed at $70{ }^{\circ} \mathrm{C}$ under an argon stream. The reaction was stop ca. $101.0 \mathrm{~h}$ later when the ratio of the integral intensities of peaks at ca.5.6-6.4 ppm (attributed to the protons in the vinyl groups) to those at ca. 4.0 ppm (of $\alpha$-carbons in the 1, 4-butanediol units) was close to 0.375 as monitored by ${ }^{1} \mathrm{H}$ NMR. After cooling down to ambient temperature, the solution was used for tuning of terminal groups directly.

In order to prepare poly(BDA2-AEPZ1)-vinyl without exposure to air, first the dissolved air in the solution of BDA and AEPZ were removed by three rounds of freeze 
vacuuming -thaw purging with argon. Then the polymerization was performed under argon flow.

\section{Attachment of the terminal groups of hyperbranched poly(amino ester)}

Poly(BDA2-AEPZ1)-OH $\left(\mathrm{M}_{\mathrm{n}}=78650, \mathrm{PDI}=1.89\right)$ The solution of poly(BDA2-AEPZ1) -vinyl in DMSO was added dropwise into a chloroform solution of AEOL. The molar ratio of AEOL to the residual vinyl groups was $10: 1$. The reaction was performed for half an hour. Then the solution was precipitated into diethyl ether under vigorous stirring. The polymer was collected and purified by precipitation from a chloroform solution into diethyl ether for three times followed by drying under vacuum at room temperature for 3 days. ${ }^{13} \mathrm{C}$ NMR $\delta$ (DMSO, 100.62 MHz) 172.72, 64.13, 60.96, 56.88, 54.10, 53.95, 53.39, $52.15,51.45,50.05,45.56,35.29,33.02,32.77,25.68$.

Poly(BDA2-AEPZ1)-(OH $)_{2}\left(\mathrm{M}_{\mathrm{n}}=87820\right.$, PDI =1.31 $)$ The solution of poly(BDA2AEPZ1)-vinyl in DMSO was added dropwise into a DMSO solution of APDIOL. The molar ratio of AEOL to the residual vinyl groups was $5: 1$. The reaction was performed for half of an hour, and the solution was precipitated into diethyl ether under vigorous stirring. The polymer was collected and purified by precipitation from a chloroform solution into diethyl ether for three times followed by drying under vacuum at room temperature for 3 days. ${ }^{13} \mathrm{C}$ NMR $\delta$ (DMSO, 100.62 MHz) 172.78, 71.26, 65.49, 64.13, $56.90,54.10,53.96,53.48,53.40,51.47,50.07,45.96,35.53,33.04,32.79,25.70$.

Poly(BDA2-AEPZ1)-NH2 The solution of poly(BDA2-AEPZ1)-vinyl in DMSO was slowly added dropwise into a DMSO solution of AEPZ under stirring. The molar ratio of AEPZ to the residual vinyl group was $5: 1$. The solution was precipitated into diethyl ether under vigorous stirring $0.5 \mathrm{~h}$ later. The polymer was collected and purified by 
precipitation from a chloroform solution into diethyl ether for three times followed by drying under vacuum at room temperature for 3 days. ${ }^{13} \mathrm{C} \mathrm{NMR} \delta\left(\mathrm{CDCl}_{3}, 100.62 \mathrm{MHz}\right)$ $172.67,64.15,61.11,56.78,53.86,53.46,53.20,51.58,49.94,38.98,32.92,32.63,25.57$. Hydrolysis profile of poly(amino ester)s The degradation of poly(amino ester)s in deuterium water solution was monitored in situ using ${ }^{1} \mathrm{H}$ NMR. After the hydrolysis, the peak attributed to the proton attached to the $\beta$-carbons in 1,4-butanediol shifted from around $1.50 \mathrm{ppm}$ to $1.37 \mathrm{ppm}$. Therefore the hydrolysis degree could be monitored by the change in the ratio of the integral intensities of the two peaks as expressed as I1.37/(I1.50 + I1.37). The hydrolysis profile of poly(BDA2-AEPZ1)-OH is illustrated in Figure S4. 


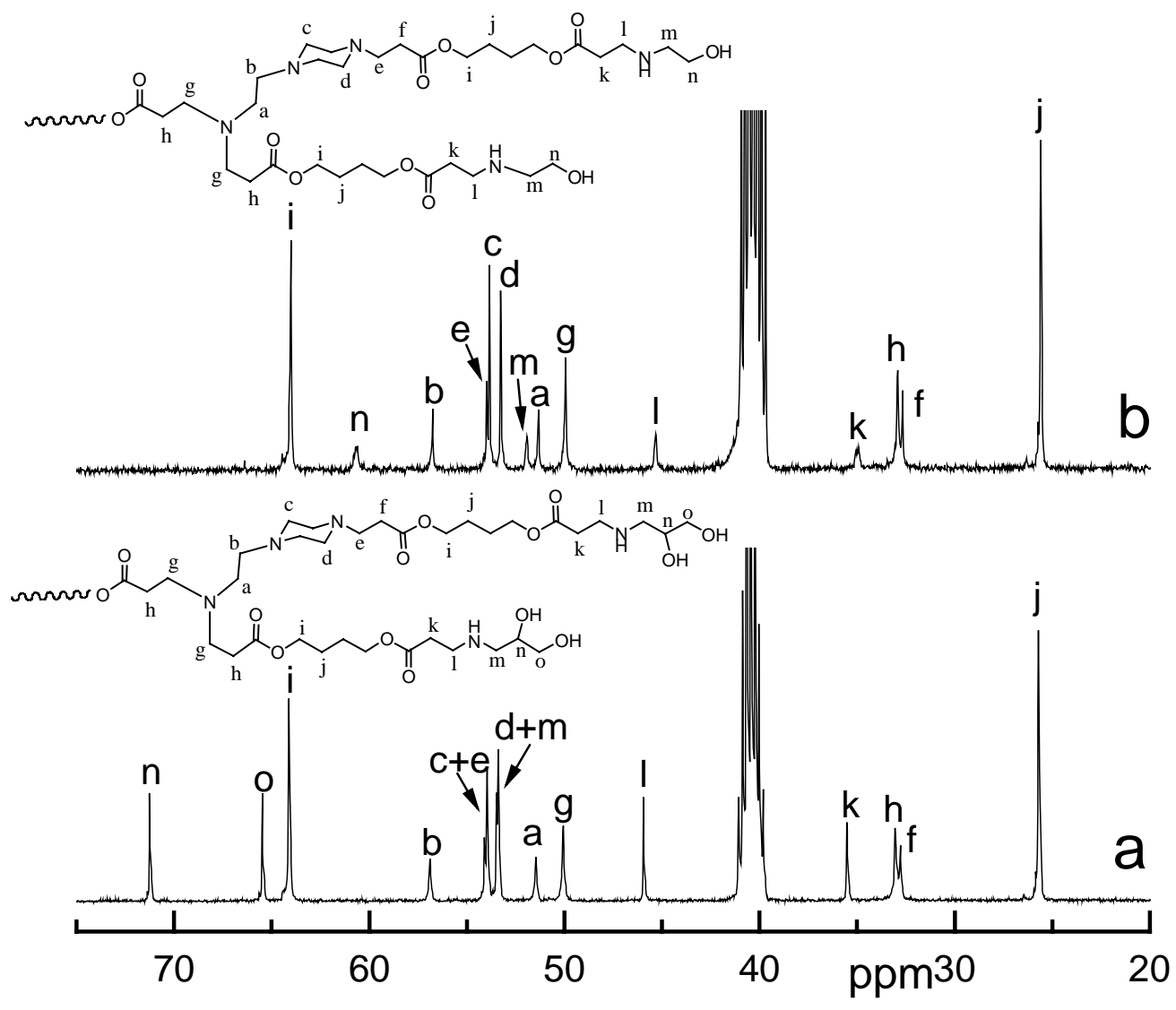

Figure S1. ${ }^{13} \mathrm{C}-\mathrm{NMR}$ spectrum of a) hyperbranched poly(BDA2-AEPZ1)-(OH $)_{2}$ and b) hyperbranched poly(BDA2-AEPZ1)-OH in DMSO- $d_{6}$. 


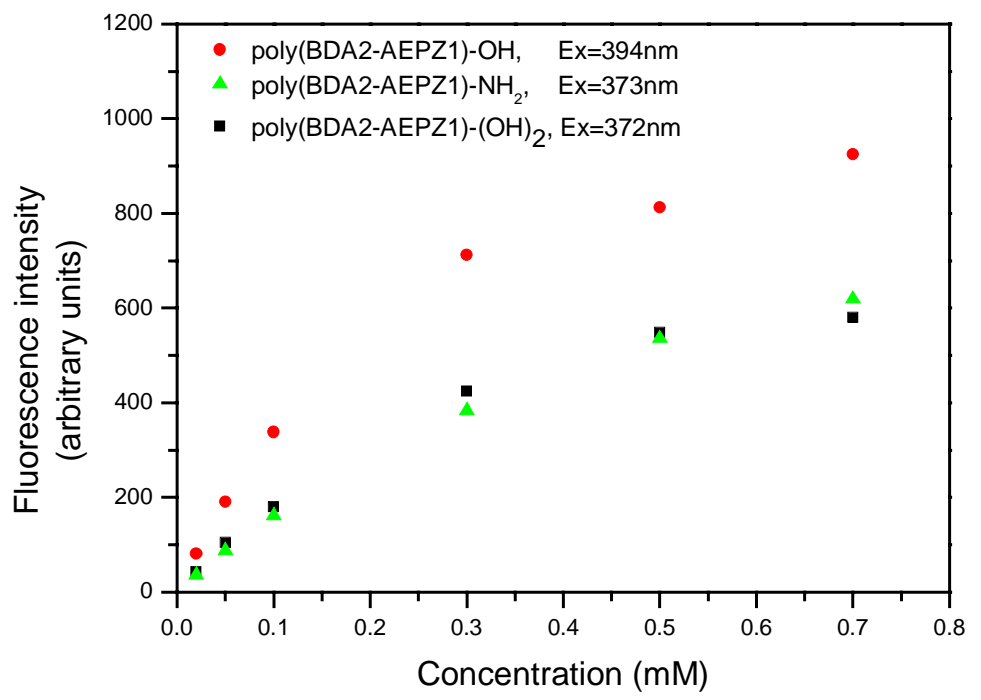

Figure S2. Fluorescence intensities of aqueous solutions of poly(BDA2-AEPZ1)-OH, poly(BDA2-AEPZ1)-NH $\mathrm{NH}_{2}$ and poly(BDA2-AEPZ1)-(OH $)_{2}$ of different concentrations at $\mathrm{pH} 7$ with exposure to air. The excitation and emission bands for poly(BDA2-AEPZ1)-OH, poly(BDA2-AEPZ1)-NH $\mathrm{NH}_{2}$ and poly(BDA2-AEPZ1)-(OH) $)_{2}$ were at 394, 373 and $372 \mathrm{~nm}$ and 473, 469 and $456 \mathrm{~nm}$, respectively. 


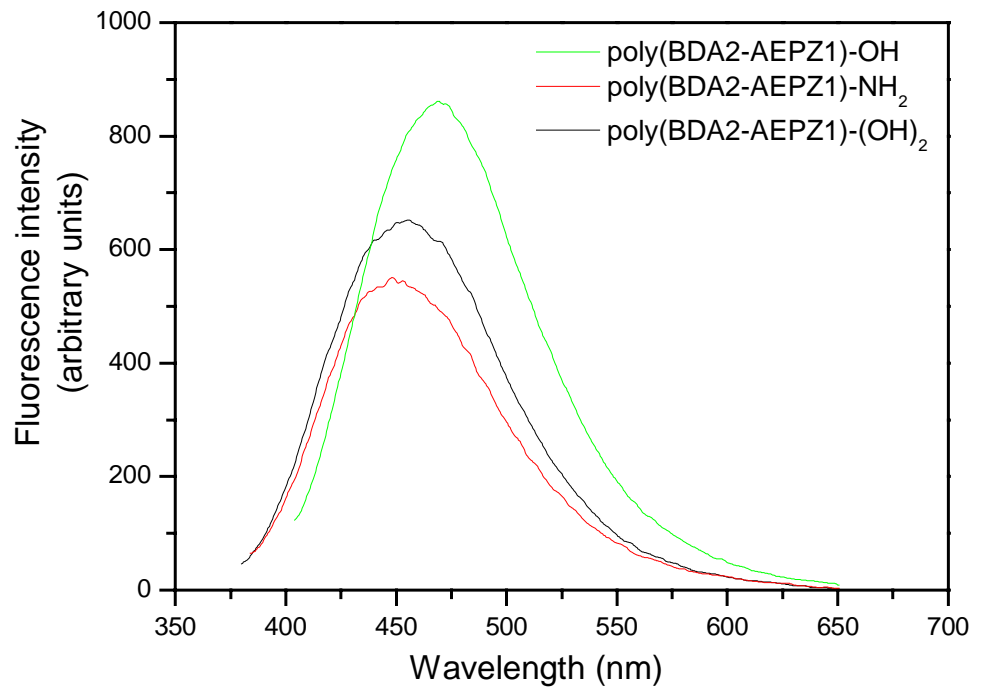

Figure S3. Emission spectra of $0.5 \mathrm{mM}$ solution poly(BDA2-AEPZ1)-OH, poly(BDA2AEPZ1)- $\mathrm{NH}_{2}$ and poly(BDA2-AEPZ1)-(OH $)_{2}$ in THF with $394 \square 373$ and 372 nm excitation, respectively, with exposure to air. 


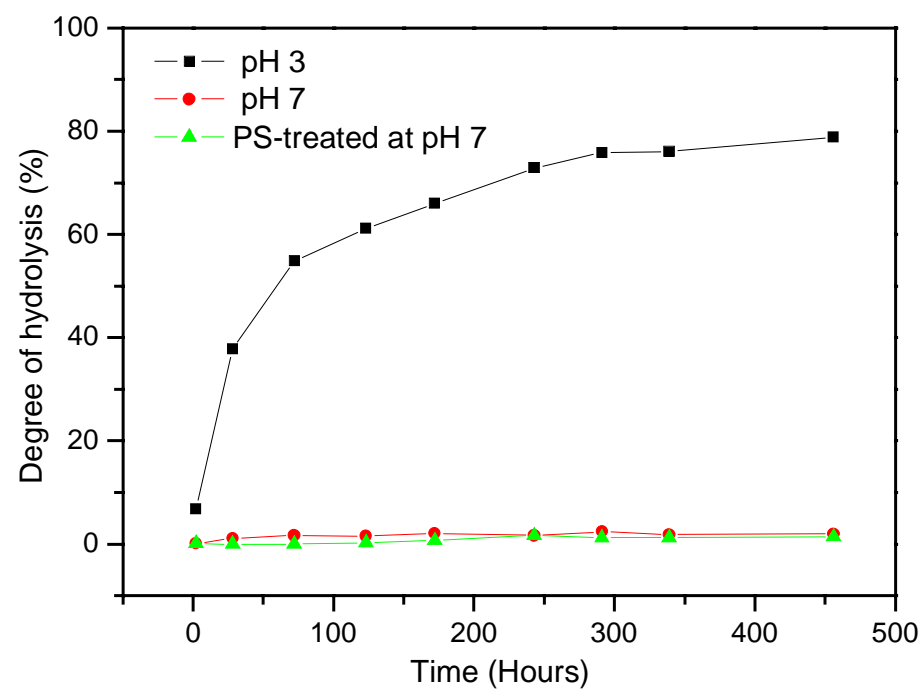

Figure S4. Hydrolysis profile of poly(BDA2-AEPZ1)-OH under different $\mathrm{pH}$. 


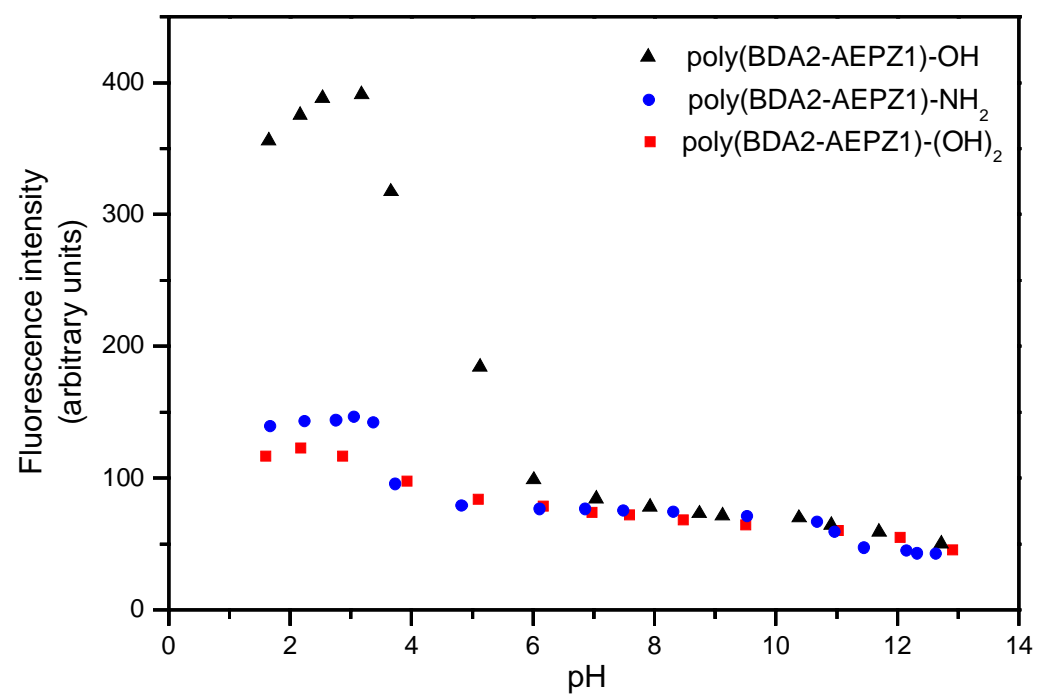

Figure S5. pH-Dependent fluorescence intensities of $0.5 \mathrm{mM}$ aqueous solutions of poly(BDA2-AEPZ1)-OH, poly(BDA2-AEPZ1)- $\mathrm{NH}_{2}$ and poly(BDA2AEPZ1)-(OH $)_{2}$ with exposure to air. The excitation and emission bands of poly(BDA2-AEPZ1)-OH, poly(BDA2-AEPZ1)- $\mathrm{NH}_{2}$ and poly(BDA2AEPZ1)-(OH $)_{2}$ were at $394,373,372 \mathrm{~nm}$ and $473,469,456 \mathrm{~nm}$, respectively. 


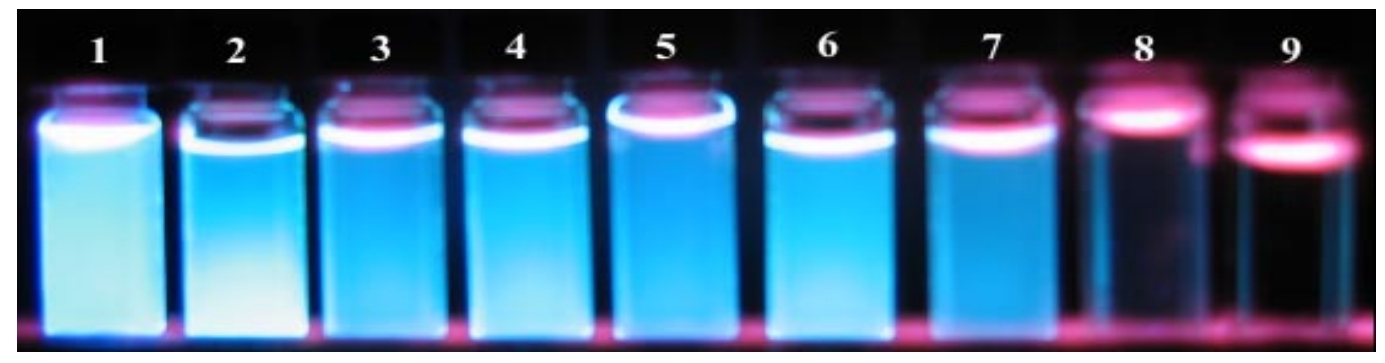

Figure S6. Illumination photographs of $0.5 \mathrm{mM}$ aqueous solutions of polymers with exposure to air. The solutions were excited under 365-nm irradiation. (1) 0.01 mM quinine sulphate; (2) poly(BDA2-AEPZ1)-OH (pH 3); (3) poly(BDA2AEPZ1)-OH (pH 7); (4) poly(BDA2-AEPZ1)-(OH)2 (pH 3); (5) poly(BDA2AEPZ1)- $(\mathrm{OH})_{2} \quad(\mathrm{pH} \quad 7) ; \quad(6) \quad \operatorname{poly}(\mathrm{BDA} 2-\mathrm{AEPZ1})-\mathrm{NH}_{2} \quad(\mathrm{pH} \quad 3) ; \quad$ (7) poly(BDA2-AEPZ1)-NH2 (pH 7); (8) poly(BDA-PZ) (pH 3); (9) PEI (25,000) (pH 3). 


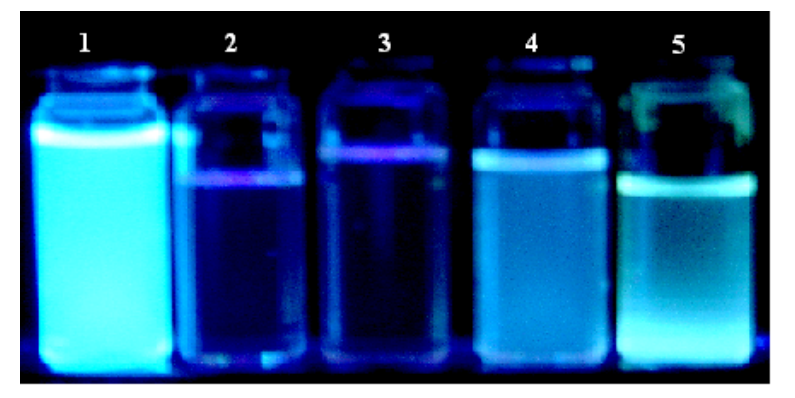

Figure S7. Comparison of the luminescent photographs of different solution. The solutions were excited under 365-nm irradiation. (1) $0.01 \mathrm{mM}$ quinine sulphate (aqueous solution); and 0.5 mM DMSO solutions of (2) BDA; (3) AEPZ; (4) 2BDA+AEPZ (just after mixing); (5) poly(BDA2-AEPZ1)-vinyl without exposure to air. 